\title{
Myeloid Toxicity of Cancer Treatment
}

\author{
SANDRA KURTIN, RN, MS, AOCN ${ }^{\circledR}$, ANP-C
}

From Arizona Cancer Center,

Tucson, Arizona

Author's disclosures of potential conflicts of interest are found at the end of this article.

Correspondence to: Sandra Kurtin, RN, MS, AOCN, ANP-C, Arizona Cancer Center, $3838 \mathrm{~N}$. Campbell Avenue, Tucson, AZ 85719. E-mail: skurtin@umcaz.edu

(c) 2012 Harborside Press ${ }^{\circledast}$

\begin{abstract}
Myelotoxicity is one of the most common treatment-related adverse events for patients receiving systemic antineoplastic therapy or radiotherapy to bone marrow-producing regions. Myeloid cytopenias, including neutropenia, thrombocytopenia, and anemia, are the most common manifestations of treatment-related myelotoxicity and one of the most common reasons for dose modifications, dose delays, or discontinuation of therapy, potentially limiting therapeutic benefit. Risk factors for myelotoxicity can be broadly categorized into three types: disease-related, host-related, and treatmentrelated. Familiarity with factors predictive of high-risk febrile neutropenia, bleeding due to thrombocytopenia, and cardiopulmonary compromise due to anemia will provide the advanced practitioner (AP) in oncology with critical tools for rapid identification of patients at risk, prompt implementation of established guidelines for management, and avoidance of clinical deterioration. The AP in oncology is often the primary point of contact for management of cytopenias, including administration of myeloid growth factors, transfusion of blood products, and management of acute events such as neutropenic fevers. Each of these interventions requires familiarity with the risk and benefits of treatment. This article will review the physiology of the bone marrow, risk factors for cytopenias, and current guidelines and recommendations for prevention and treatment of myeloid toxicity of cancer treatment.
\end{abstract}

J Adv Pract Oncol 2012;3:209-224

yelotoxicity is one of the most common treatment-related adverse events for patients receiving systemic antineoplastic therapy or radiotherapy to bone marrow-producing regions. Myeloid cytopenias-including neutropenia, thrombocytopenia, and anemia-are the most frequently seen manifestations of treatment-related myelotoxicity and one of the most common reasons for dose modifications, dose delays, or discontinuation of therapy, potentially limiting therapeutic benefit. Lymphopenia, although less common, presents unique challenges and may place the patient at increased risk for opportunistic and often life-threatening infections. Proactive management of cytopenias can improve treatment tolerance and treatment outcomes. An understanding of 
the physiology of the bone marrow, normal hematopoiesis, risk factors for treatment-related cytopenias, strategies for minimizing serious adverse events (AEs), and adaptation and consistent application of these concepts for individual patient populations will limit the severity of hematologic AEs and improve treatment outcomes.

The advanced practitioner (AP) in oncology is often the primary point of contact for management of cytopenias, including administration of myeloid growth factors, transfusion of blood products, and management of acute events such as neutropenic fever. The American Society of Clinical Oncology (ASCO), the National Comprehensive Cancer Network (NCCN), the American Society of Hematology (ASH), and the Multinational Association for Supportive Care in Cancer (MASCC) have published recommendations or guidelines for the management of treatment-related cytopenias. The US Food and Drug Administration (FDA) and the American Association of Blood Banks (AABB) have established guidelines for the administration of hematopoietic growth factors and blood products. Familiarity with these guidelines and recommendations, together with a working knowledge of common disease- and treatment-related risk fac- tors, will provide a sound foundation for effective management of treatment-related myelotoxicity.

This article will focus on the clinical management of treatment-related myeloid cytopenias, including current guidelines and recommendations from the societies and associations noted above. Lymphopenia and the management of common infectious complications were previously discussed in JADPRO's series of articles on treatment-related adverse events (Wood \& Payne, 2011).

\section{Bone Marrow Physiology and Normal Hematopoiesis}

The bone marrow is the primary source for development of the components of blood (hematopoiesis), including myeloid and lymphoid progenitor cells (Figure 1). Hematopoiesis occurs primarily in the axial skeleton, with the majority of production taking place in the pelvis $(70 \%-$ $72 \%$ ), the long bones such as the femurs, the skull, the sternum, the ribs, and vertebral bodies (Gatter, Natkunam, \& Brown, 2008). Extramedullary hematopoiesis, or production of the elements of blood outside the bone marrow, may occur in the spleen and other accessory sites in selected disease states such as the myeloproliferative disorders and chronic leukemia (Kurtin, 2011b). The

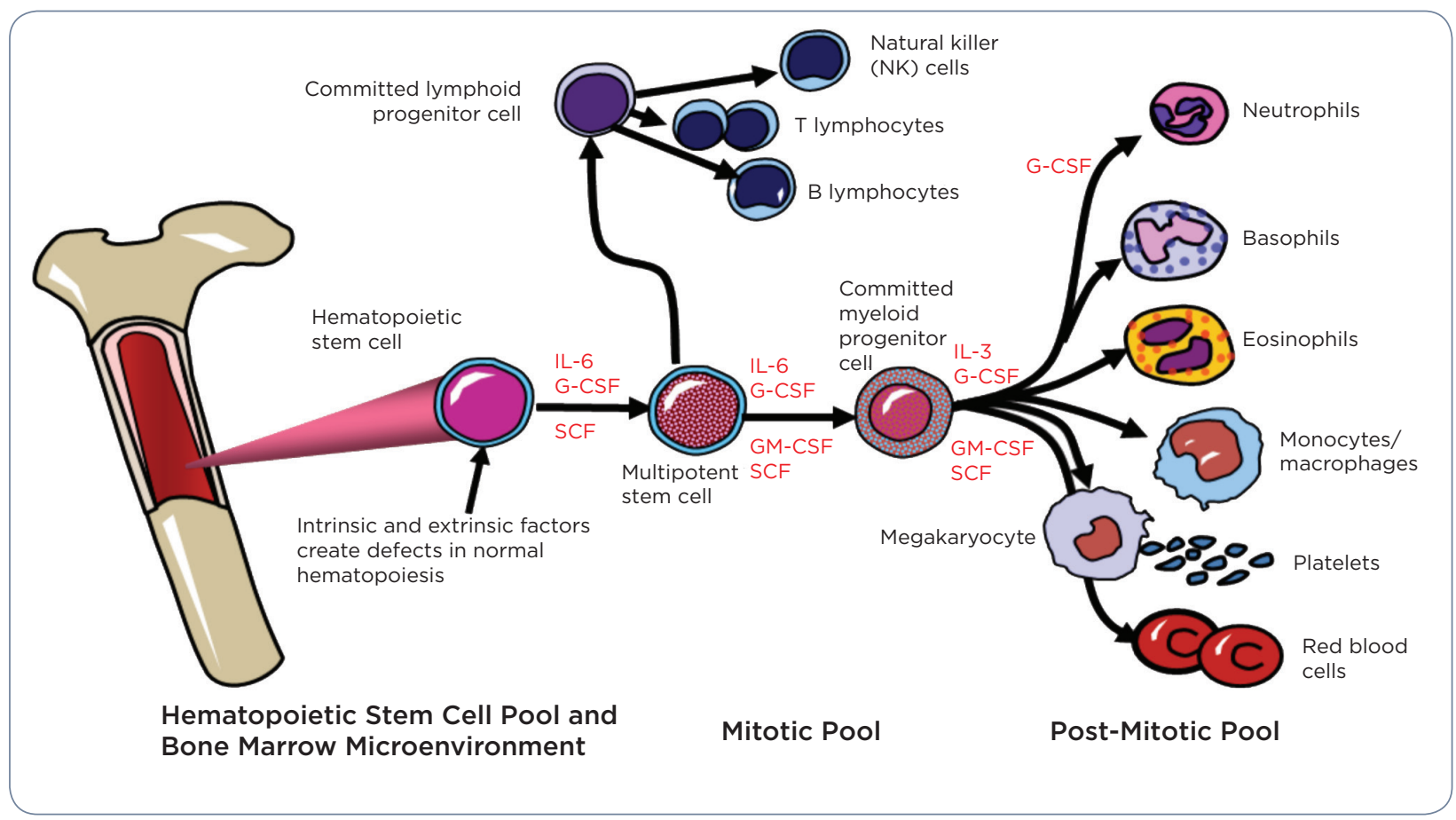

Figure 1. Bone marrow physiology. G-CSF = granulocyte colony-stimulating factor; GM-CSF = granulocyte-macrophage colony-stimulating factor; IL = interleukin; SCF = stem cell factor. Adapted from NIH (2012), Metcalf (2010), Crea et al. (2009), and Kurtin (2011a). 
bone marrow comprises trabecular bone, stromal elements, hematopoietic cells, and elements of the bone marrow microenvironment (Table 1). Each of these elements plays a role in normal or abnormal hematopoiesis and represents a different percentage of the bone marrow space, in part determined by age and underlying disease.

Normal myeloid hematopoietic progenitor cells arise from multipotent hematopoietic stem cells (HSCs) that are capable of self-renewal, allowing for continuous replacement of granulocytes, macrophages, and erythrocytes. The majority of HSCs remain noncycling, with only a few active at any time for release of these early progenitor cells (Crea et al., 2009). In the presence of more severe hematopoietic stress, such as bleeding, infection, or chemotherapy exposure, a greater number of HSCs are activated to increase progenitor production (Crea et al., 2009). The normal life cycle for each cell type varies. However, the continuous renewal and life cycle of the myeloid lineage is rapid compared with other tissue cell lines, playing a primary role in susceptibility to the effects of chemotherapeutic agents (Gatter et al., 2008; Maxwell \& Maher, 1992).

The capacity for self-renewal is regulated by a number of intrinsic and extrinsic factors, including genetic and molecular mechanisms, cytokines, and signaling pathways (Corey et al., 2007; Bejar, Levine, \& Ebert, 2011; Kurtin, 2011a); see Table 1 . The process of apoptosis plays a primary role in regulating the "on/off" mechanism of the self-renewal process. Defects in each cell line and in the microenvironment, including apoptosis, play a key role in the pathobiology of hematologic malignancies and contribute to susceptibility to cytopenias, the capacity for hematopoietic recovery, and the abnormal findings in the bone marrow or peripheral blood (Gatter et al., 2008; Carlesso \& Cardoso, 2010; Crea et al., 2009). Patients receiving antineoplastic therapies for solid tumors may develop treatment-induced abnormalities, causing similar changes and contributing to cytopenias, although the severity and duration of cytopenias are generally less severe.

The concept of the stem cell niche, highly specialized bone marrow compartments with intricate regulatory processes driving stem cell development and maturation, is an evolving idea in the understanding of normal and malignant hematopoiesis (Carlesso \& Cardoso, 2010). These discov- eries have elucidated new areas for investigating the prognostic significance of cytopenias at diagnosis and during treatment and the potential for off-target effects of growth factor administration.

Clinical evaluation of bone marrow function relies on analysis of peripheral blood (postmitotic pool) and a bone marrow biopsy and aspirate (mitotic pool/progenitor pool); see Figure 1 and Table 1. A complete blood count with differential and platelet count is the most common method for analysis of the postmitotic pool and will reflect the end results of bone marrow production with any secondary effects of the host. Bone marrow analysis is required for the diagnosis and risk analysis for hematopoietic malignancies, including cytogenetic and molecular information necessary for prognostication. The most common site for obtaining bone marrow samples is the posterior iliac crest, due to the accessibility, relative low risk, and bone marrow-producing capacity. The anterior iliac crest may also be used for sampling, although this is generally more difficult due to the architecture, accessibility, and depth of the cortical bone.

Bone marrow aspirates may be obtained from the sternum using a specialized needle with a shield if there are contraindications for pelvic biopsies, such as for those patients who are intubated and are difficult to turn, for those with a fractured pelvis, or for patients who have had extensive radiotherapy to the pelvis, which may limit the diagnostic value of the specimen. Core biopsies may not be obtained from the sternum due to a high risk of sternal fracture and penetration of surrounding structures with potential for fatal hemorrhage, limiting the diagnostic evaluation. Bone marrow evaluation is performed infrequently in patients with solid tumors unless there are concerns for abnormal findings in the peripheral blood. Adequate bone marrow samples are necessary to provide a complete analysis of the mitotic pool, postmitotic pool, and disease attributes including genetic or molecular features. A

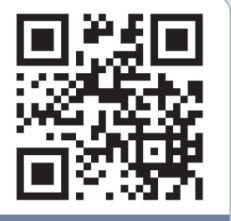

Use your smartphone to access the Common Terminology Criteria for Adverse Events (CTCAE) from the U.S. Department of Health \& Human Services.

SEE PAGE 272 
Table 1. Key Elements of the Myeloid Lineage

\section{Element \\ Components, features, and clinical significance}

Bone marrow, mitotic pool: Intrinsic features

Hematopoietic stem cells

Multipotent with self-renewal capacity, few in active division Insensitive to standard chemotherapy or growth factors Cytokine sensitivity: G-CSF, SCF, IL-6

Myeloid progenitor cells

Committed to myeloid lineage, CFU-GEMM, differentiates into postmitotic pool: erythroid, granulocyte/macrophage, and megakaryocytic/platelet cell lines. CD34+ cells stimulated and collected for stem cell transplantation. Cytokine sensitivity: G-CSF, SCF, IL-6, EPO

Cytogenetics and molecular Chromosomal and molecular attributes have prognostic significance in all attributes hematologic malignancies and play a primary role in abnormal clonal evolution and ineffective hematopoiesis.

Bone marrow microenvironment (HSC niche): Extrinsic features

Trabecular bone

Rich in osteolineage cells (osteoblasts and osteoclasts), which play a role in regulation of HSC activity, mediated by cytokines active in the stroma and cytokine milieu (in particular, erythropoietin and IL-6) as well as parathyroid hormone and TNF $\alpha$. Mesenchymal cells may play a role in the nervous system regulation of hematopoiesis. Abnormal characteristics may contribute to tumorigenesis.

Stroma (bone marrow vascular niche)

Cytokine milieu

\section{Component}

Peripheral blood, postmitotic pool (myeloid lineage) ${ }^{a}$

Erythrocytes

Platelets lifespan 120 days

Reticular perivascular cells, reticulin fibers, fibroblasts, adhesion molecules, adipocytes, iron stores. Rich in cytokines and hormones such as VEGF and TNF $\alpha$, which play a role in regulating hematopoiesis. Sensitive to paracrine- and autocrine-mediated cytokines as well as exogenous exposure to cytokines and stress hormones. Abnormal characteristics may contribute to tumorigenesis and may serve as a sanctuary site for leukemic stem cells.

Glycoproteins that bind to cell surface receptors; includes IL-3, IL-6, SCF, G-CSF, EPO, TPO

\section{Normal range and life cycle}

Total WBCs

Neutrophils

Monocytes

Eosinophils

Basophils

Note. G-CSF = granulocyte colony-stimulating factor; SCF = stem cell factor; IL-6 = interleukin-6; CFU-GEMM = colonyforming unit-granulocyte erythrocyte macrophage monocyte; EPO = erythropoietin; HSC = hematopoietic stem cells; TNF $\alpha$ = tumor necrosis factor-alpha; VEGF = vascular endothelial growth factor; IL-3 = interleukin-3; TPO = thrombopoietin; $\mathrm{Hgb}=$ hemoglobin; $\mathrm{Hct}=$ hematocrit; $\mathrm{WBCs}=$ white blood cells. Information from Carlesso \& Cardoso (2010), Singbrant et al. (2011), Metcalf (2010), and Crea et al. (2009).

${ }^{a}$ General attributes: Low sensitivity to chemotherapy; high sensitivity to G-CSF, EPO, and TPO

summary of the key elements of the bone marrow with normal variants and life cycles for individual cell lines is included in Table 1.

\section{Risk Factors for Myelotoxicity}

Risk factors for myelotoxicity can be broadly categorized into three basic types: disease-related, host-related, and treatment-related. Disease-related factors can be further divided into solid tumors vs. hematologic malignancies. The incidence, severity, and duration of myeloid cytopenias are greatest in the hematologic malignancies. Cytopenias may be present at the time of diagnosis as a result of abnormal cellular development or bone marrow 
infiltration. In some cases, such as chronic lymphocytic leukemia, autoimmune processes such as hemolytic anemia or idiopathic thrombocytopenia purpura may be present at diagnosis, contributing to the abrupt onset or severity of anemia or thrombocytopenia. Patients with massive splenomegaly due to acute or chronic lymphocytic leukemia or myeloproliferative disorders may present with thrombocytopenia due to splenic sequestration or peripheral destruction of platelets. Thus, evaluation of tumor burden at the time of diagnosis in patients with hematologic malignancies is a critical first step in estimating the risk for cytopenias with initiation of treatment. Furthermore, determining the extent of risk will assist in setting the expectations for both the patient and the providers as treatment is initiated.

It is not uncommon to expect moderate to severe cytopenias in the early phases of treatment for hematologic malignancies, as the primary target for treatment resides in the bone marrow, and both normal and abnormal cells will be affected. Understanding the concepts of expected cytopenias, cytopenias getting worse before they get better, and sustained moderate but asymptomatic cytopenias is critical for the AP in oncology managing patients with hematologic malignancies (Kurtin, Demakos, Hayden, \& Boglione, 2012; Kurtin, 2011a). Treatmentrelated cytopenias are much less common in patients with solid tumors. However, these patients may also experience moderate to severe myeloid cytopenias as a result of bone marrow infiltration by tumor, radiation to bone marrowproducing sites, and other treatment- or hostrelated factors.
The bone marrow's capacity to recover is an important consideration in all patients. Patients with limited cellularity, extensive bone marrow fibrosis, underlying aplasias, or treatment-related secondary malignancies have an impaired ability to recover normal hematopoiesis. In most cases, the only option for restoration of normal hematopoiesis is an allogeneic hematopoietic stem cell transplant, which is limited primarily to patients with hematologic malignancies who meet stringent transplant eligibility criteria, including adequate organ function, a suitable donor, and the availability of a consistent caregiver.

\section{Host-Related Factors}

In addition to disease-related factors, selected attributes of the individual patient may increase the risk of treatment-related cytopenias (Table 2). Hematopoietic senescence is common in older patients due to the normal functional decline of the bone marrow with increasing age (Kurtin, 2010). The bone marrow of an older adult is generally less cellular (estimated as bone marrow cellularity $\%=100-$ patient age in years) with a higher fat content, increasing the susceptibility to cytopenias. Older patients more often experience compromised renal or hepatic function, which may contribute to cytopenias due to impaired metabolism of chemotherapeutic agents (Scripture \& Figg, 2006; Kurtin, 2010). Dose modification is required for selected chemotherapeutic agents in the instance of renal or hepatic impairment to limit the severity of AEs, including cytopenias. Similarly, comorbid conditions and associated medications may contribute to decreased bone marrow function

Table 2. Factors Associated With High Risk for Chemotherapy-Induced Myelotoxicity

\author{
Host-related factors \\ Age $>65 \mathrm{yr}$ \\ Female gender \\ ECOG PS $>1$ \\ Malnutrition \\ Immunosuppression \\ Comorbidities: COPD, diabetes, renal impairment, \\ liver disease \\ Open wounds or recent surgery \\ Active infection or preexisting fungal infections \\ Drug-drug interactions
}

\section{Disease- and treatment-related factors}

High tumor burden/extensive disease History of chemotherapy or radiation Preexisting cytopenias

Bone marrow involvement with tumor

Type of chemotherapy

Dose intensity of chemotherapy

Elevated lactate dehydrogenase level

Hypoalbuminemia

Hyperbilirubinemia, hematologic malignancy,

hospitalization

Note. ECOG = Eastern Cooperative Oncology Group; PS = performance status; COPD = chronic obstructive pulmonary disease. Information from Scripture \& Figg (2006), Daniel \& Crawford (2006), Aapro et al. (2011), and Schwenkglenks et al. (2011). 
or increased risk of drug interactions and treatment-related AEs (Carreca \& Balducci, 2009; Scripture \& Figg, 2006).

Certain drugs are known to be associated with cytopenias independent of bone marrow function, including immunosuppressive agents, anti-inflammatory medications, and antibiotics. Careful review of medications that may contribute to cytopenias is necessary to limit the severity of AEs and to avoid unnecessary dose modification of antineoplastic therapies. Malnutrition affects many cellular processes, including hematopoiesis. Decreased serum albumin levels may indicate an increased risk for treatment-associated AEs and are included in the risk analysis for many hematologic malignancies (Kurtin, 2010; Greipp et al., 2005).

\section{Treatment-Related Myelotoxicity}

Chemotherapy-induced myelosuppression is the most common dose-limiting AE for patients receiving cancer treatment. The incidence, severity, and duration of myelosuppression vary by drug, dependent on pharmacokinetic variables of dose, frequency, route of administration, absorption, distribution, metabolism, and excretion (Undevia, Gomez-Abuin, \& Ratain, 2005). Each antineoplastic agent varies with respect to the onset and duration of cytopenias. In general, cytopenias are dose dependent, so dose reductions or delays may be effective in minimizing the severity of cytopenias but may also limit the therapeutic potential of treatment. Dose-intensity and combination therapies are the most common approach used in cancer treatment regimens with the intent to exploit different mechanisms of action and vulnerabilities of the tumor while balancing toxicity profiles. However, increased doses and combination therapies frequently increase the potential for cytopenias (Crawford et al., 2011; Smith, 2006). Several guidelines and recommendations have been proposed by international oncology organizations to estimate the risk for selected cytopenias and to provide evidence-based treatment guidelines (Table 3).

The combination of chemotherapy and radiation therapy is commonly used to treat esophageal, gastric, head and neck, rectal, anal, and pancreatic tumors. Each of these disease states may include areas of bone marrow-producing regions in the radiation field, increasing the potential for more severe and sustained cytopenias, complicating the delivery of systemic therapy during or following chemoradiotherapy (Chan et al., 2011; Miyoshi et al., 2009). Administration of myeloid growth factors during radiation therapy is generally avoided in these instances, as these agents promote cell division, increasing the potential myelosuppressive effect. Patients receiving treatment over extended periods of time, such as those with metastatic disease, may develop cumulative myelotoxicity and are at risk for secondary malignancies including acute myeloid leukemia (AML) and myelodysplastic syndromes (MDS). The onset of AML and MDS varies according to the agents administered, with early onset (within 3 years) for patients receiving topoisomerase II inhibitors such as etoposide, teniposide, topotecan, and doxorubicin, and late onset (5 to 10 years) for patients receiving radiation or therapeutic alkylators such as cyclophosphamide (Sekeres, 2011; Kurtin, 2011b). Chromosome 5 or 7 abnormalities are most common in these patients and are associated with a poor prognosis.

The late onset of cytopenias, progressive cytopenias unexplained by ongoing treatment, the presence of circulating blasts, or the onset of pancytopenia in cancer survivors will require a bone marrow biopsy and aspirate to further characterize the cause. Specimens should be sent for hematopathology, flow cytometry, and cytogenetic analysis to provide the necessary diagnostic information.

\section{Clinical Implications of Neutropenia}

Chemotherapy-induced neutropenia (CIN) is one of the most common dose-limiting toxicities associated with systemic treatment for cancer due to cytotoxic effects to the rapidly dividing neutrophils, as well as damage to elements of the stroma and cytokine milieu. Mature granulocytes, including neutrophils, have a lifespan of 1 to 3 days, thus they have a rapid mitotic rate and greater susceptibility to cytotoxic damage than other myeloid cell lines with longer lifespans (platelets $~ 10-12$ days, erythrocytes $~ 120$ days; Crea et al., 2009). The onset and duration of neutropenia vary widely by agent, dose, frequency of dosing, and host-related factors previously discussed. Neutrophil precursors are more prevalent than erythroid or platelet cell lines, accounting for more than $50 \%$ of the hematopoietic cells in the bone marrow and postmitotic pool, with only $2 \%$ in circulation and $3 \%$ in the spleen or vascula- 
Table 3. Common Chemotherapeutic Regimens for Selected Tumor Types With Intermediate to High Risk for Myelotoxicity ${ }^{a}$

Bladder cancer

MVAC: methotrexate, vinblastine, doxorubicin, cisplatin $(\mathrm{H})$

Breast cancer

$A C \rightarrow T$ with trastuzumab (I)

CMF: cyclophosphamide, methotrexate, 5-FU (I)

Docetaxel every 21 days (I)

Epirubicin as a single agent or in sequential regimens with cyclophosphamide, 5-FU, methotrexate (I)

FEC $\rightarrow$ T: 5-FU, epirubicin, cyclophosphamide, and sequential docetaxel (I)

Paclitaxel every 21 days (I)

AT: doxorubicin/paclitaxel $(H)$

Dose-dense $A C \rightarrow T$ : doxorubicin, cyclophosphamide, paclitaxel $(\mathrm{H})$

Docetaxel/trastuzumab $(\mathrm{H})$

TAC: docetaxel, doxorubicin, cyclophosphamide $(\mathrm{H})$

Paclitaxel/lapatinib (I)

Vinblastine (I)

\section{Cervical cancer}

Cisplatin/topotecan (I)

Topotecan (I)

Irinotecan (I)

\section{Colorectal cancer}

FOLFOX: 5-FU, leucovorin, oxaliplatin (I)

Esophageal and gastric cancers

DCF: docetaxel, cisplatin, 5-FU (H)

Irinotecan/cisplatin (I)

ECF: epirubicin, cisplatin, 5-FU (I)

Hodgkin lymphoma

ABVD: doxorubicin, bleomycin, vinblastine, dacarbazine (I)

BEACOPP: bleomycin, etoposide, doxorubicin, cyclophosphamide, vincristine, procarbazine, prednisone $(\mathrm{H})$

Stanford V: mechlorethamine, doxorubicin, vinblastine, bleomycin, etoposide, prednisone (I)

Kidney cancer

Doxorubicin/gemcitabine $(H)$

\section{Melanoma}

Dacarbazine-based combinations with or without IL-2 (H)

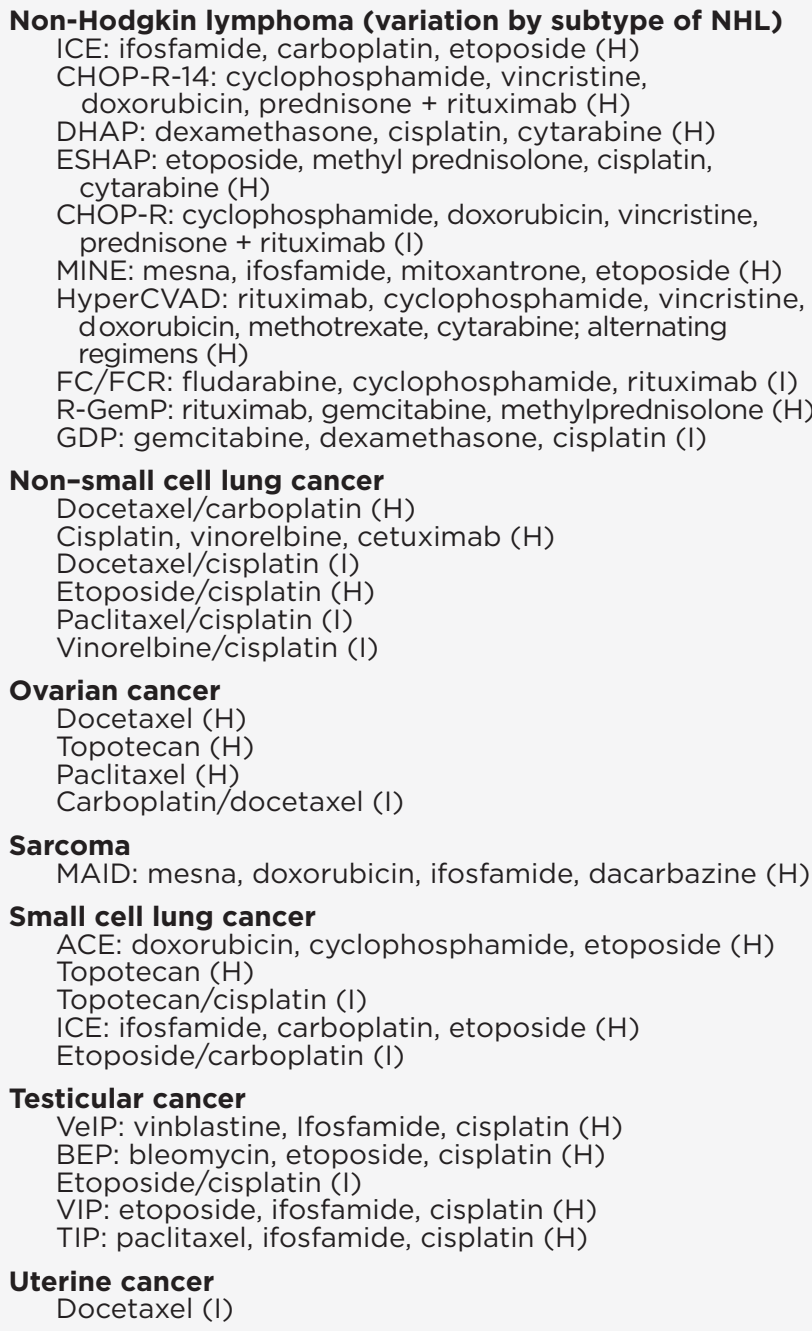

Note. I = intermediate risk; $\mathrm{H}$ = high risk; 5-FU = fluorouracil; IL-2 = interleukin 2; NHL = non-Hodgkin Iymphoma. Information from Chan et al. (2011), Crawford et al. (2011), and Aapro et al. (2011).

antermediate risk: 10\%-20\%; high risk: > 20\%. Excludes myeloid malignancies and multiple myeloma.

ture (Crea et al., 2009).

The actively dividing cells in the mitotic pool and postmitotic maturation pool are the most sensitive to the effects of chemotherapy, whereas mature and fully differentiated cells in the peripheral blood are less sensitive. The degree of sensitivity for different cells in the maturation process, together with the lifespan of each cell line, helps to explain the time of onset and recovery of cytopenias and the principles of growth factor administration for treatment.

The presence of neutropenia predisposes patients to infection. The severity and duration of neutropenia, together with host-related factors and secondary effects of the treatment regimen, contribute to the risk of more serious AEs, including neutropenic fevers and bacteremia. The greatest risk of severe CIN, including febrile neutropenia (FN), is in the first cycle of chemotherapy (Klastersky, Awada, Paesmans, \& Aoun, 2010; Aapro, Crawford, \& Kamioner, 2011; Wingard \& Elmongy, 2009). As a result, the prophylactic use of colony-stimulating factors is recommended when the risk of National Cancer Institute Common Terminology Criteria for Adverse Events (CTCAE) grades $3 / 4$ CIN or FN is greater than $20 \%$ in the setting of potentially curable disease where dose intensity is neces- 
sary for optimal clinical outcomes (Crawford et al., 2011; Wingard \& Elmongy, 2009); see Table 3. Additional parameters are suggested for patients with $<20 \%$ risk of CTCAE grades $3 / 4$ CIN or FN; see Table 4.

Chan and colleagues (2011) conducted a systematic literature review to evaluate the incidence, patient characteristics, severity, supportive care strategies, and clinical impact of grades $3 / 4$ CIN for patients enrolled in randomized phase III clinical trials evaluating emerging regimens for the treatment of common solid tumors (Chan et al., 2011). A total of 1,522 randomized, controlled trials for breast, lung, ovarian, colorectal, or renal cell carcinomas were evaluated for the inclusion of criteria for reporting, grading, and management of clinically significant CIN. Only 264 of the published trials reported the incidence of grades $3 / 4$ CIN (72\%) or FN (53\%) overall. The incidence by tumor type for grades $3 / 4$ CIN or FN was most common in the studies for lung ( $\mathrm{n}=89: 82 \%, 60 \%)$, ovarian $(\mathrm{n}=25: 80 \%$, $52 \%)$, and breast ( $\mathrm{n}=73: 70 \%, 55 \%)$ cancer, with fewer cases reported for colorectal $(n=63: 60 \%$, $53 \%)$ and renal cell $(\mathrm{n}=14: 57 \%, 21 \%)$ cancers.

Only $73 \%$ of the trials included descriptions of strategies for management of these events. The most commonly reported strategies for management included dose delays (36\%), dose reductions (48\%), and granulocyte colony-stimulating factor (G-CSF) administration. The discussion of $\mathrm{G}-\mathrm{CSF}$ administration in the methods (38\%) or results (19\%) segment for each published study was rare. The authors note that this as a major limitation for application of the trial results to the general oncology population (Chan et al., 2011). Given the well-established clinical guidelines for the management and prevention of CIN and FN, inclusion of these strategies in the design and reporting for clinical trials for emerging therapies is critical to provide oncology clinicians with the data necessary to safely integrate these newer therapies into clinical practice, limit the severity of AEs, and provide patients with the most effective clinical outcomes.

Patients with FN may deteriorate rapidly. Prompt management of these patients is essential to avoid more severe AEs such as sepsis syndrome, circulatory collapse, acute respiratory failure, or death. Familiarity with factors predictive of high-risk FN will provide the AP in oncology with critical tools for rapid identification of

\section{Table 4. National Cancer Institute Common Terminology Criteria for Adverse Events Version 4: Myelotoxicity}

\begin{tabular}{|c|c|c|}
\hline Myelotoxicity & Definition & Grading \\
\hline Anemia & $\begin{array}{l}\text { A disorder characterized by a reduction in the } \\
\text { amount of hemoglobin in } 100 \mathrm{~mL} \text { of blood }\end{array}$ & $\begin{array}{l}\text { Grade } 1: \mathrm{Hgb}<\mathrm{LLN}-10.0 \mathrm{~g} / \mathrm{dL} \\
\text { Grade } 2: \mathrm{Hgb}<10.0-8.0 \mathrm{~g} / \mathrm{dL} \\
\text { Grade } 3: \mathrm{Hgb}<8.0 \mathrm{~g} / \mathrm{dL} \text {, transfusion } \\
\quad \text { indicated } \\
\text { Grade } 4: \mathrm{Hgb}<6.2-4.9 \mathrm{~g} / \mathrm{dL} \text {, life- } \\
\quad \text { threatening consequences, urgent } \\
\quad \text { intervention indicated }\end{array}$ \\
\hline Neutropenia & $\begin{array}{l}\text { A finding based on laboratory test results } \\
\text { that indicates a decrease in the number of } \\
\text { neutrophils in a blood specimen }\end{array}$ & $\begin{array}{l}\text { Grade 1: ANC }<\mathrm{LLN}-1,500 / \mu \mathrm{L} \\
\text { Grade 2: ANC }<1,500-1,000 / \mu \mathrm{L} \\
\text { Grade 3: ANC }<1,000-500 / \mu \mathrm{L} \\
\text { Grade 4: ANC }<500 / \mu \mathrm{L}\end{array}$ \\
\hline Febrile neutropenia & $\begin{array}{l}\text { A disorder characterized by an } \mathrm{ANC}<1,000 / \mu \mathrm{L} \\
\text { and a single temperature of }>38.3^{\circ} \mathrm{C}\left(101^{\circ} \mathrm{F}\right) \text { or } \\
\text { a sustained temperature of } \geq 38^{\circ} \mathrm{C}\left(100.4^{\circ} \mathrm{F}\right) \text { for } \\
\text { more than } 1 \mathrm{hr}\end{array}$ & $\begin{array}{l}\text { Grade } 3 \text { : ANC }<1,000 / \mu \mathrm{L} \text { with a single } \\
\text { temperature of }>38.3^{\circ} \mathrm{C}\left(101^{\circ} \mathrm{F}\right) \text { or } \\
\text { a sustained temperature of } \geq 38^{\circ} \mathrm{C} \\
\left.\text { (100. } 4^{\circ} \mathrm{F}\right) \text { for more than } 1 \mathrm{hr} \\
\text { Grade } 4 \text { : Life-threatening consequences, } \\
\text { urgent intervention indicated }\end{array}$ \\
\hline Thrombocytopenia & $\begin{array}{l}\text { A finding based on laboratory test results that } \\
\text { indicates a decrease in the number of platelets } \\
\text { in a blood specimen }\end{array}$ & $\begin{array}{l}\text { Grade 1: PIt }<\mathrm{LLN}-75,000 / \mu \mathrm{L} \\
\text { Grade 2: PIt }<75,000-50,000 / \mu \mathrm{L} \\
\text { Grade 3: } \mathrm{Plt}<50,000-25,000 / \mu \mathrm{L} \\
\text { Grade 4: } \mathrm{Plt}<25,000 / \mu \mathrm{L}\end{array}$ \\
\hline
\end{tabular}


these patients, prompt implementation of established guidelines for management, and avoidance of clinical deterioration (Table 5).

Patients with CIN or FN generally present with fever and/or shaking chills. Low-risk patients may be asymptomatic and found to be neutropenic on routine laboratory evaluation. The general principles of treatment for a patient with grades $3 / 4$ CIN or FN include rapidly assessing the level of risk for deterioration and stabilizing the patient if necessary, while simultaneously obtaining additional laboratory measures, including blood cultures and urinalysis for culture and sensitivity. Prompt implementation of institutional guidelines or standing orders for the management of neutropenic fevers, including antibiotic administration, is critical to prevent more serious complications.

Park and colleagues (2010) evaluated 259 episodes of $\mathrm{FN}$ in patients with hematologic malignancies $(n=137$; median age 48 , range $18-85$ years). The most common organisms isolated were gram-negative bacteria (Escherichia coli most common, 50 episodes) and gram-positive bacteria (Staphylococcus aureus most common, 22 episodes). The respiratory tract (69 episodes, $26.6 \%$ ), indwelling catheters (22 episodes, $8.5 \%$ ), and the gastrointestinal tract (20 episodes, $7.7 \%$ ) were the most common documented sites of infection (Park et al., 2010). Of the 259 episodes evaluated, 43\% (107 episodes) were documented as fever of unknown origin.

In a second trial, Klastersky and colleagues (2007) applied the MASCC risk criteria (Table 5) to a population of 2,142 cancer patients with solid tumors and hematologic malignancies receiving chemotherapy to evaluate the incidence of bacteremia in patients experiencing FN. Fifty-eight percent of the patients were considered to be at low risk for complications related to $\mathrm{FN}$. A total of 499 patients ( $23 \%$; median age 52 years) developed documented bacteremia. Gram-positive bacteremia was most common (57\%), with gram-negative (23\%) and polymicrobial bacteremia (10\%) less common. A MASCC risk score of $<15$ was associated with the poorest prognosis $(p<.001)$.

These trials emphasize the increased risk for CIN and FN in patients with hematologic malignancies, the common sources of infection, and the most common microbes isolated, providing the AP in oncology with useful information for evaluating risk and identifying treatment strategies.

\section{Table 5. Factors Associated With Poor- Prognosis Febrile Neutropenia}

Factors predictive of poor prognosis at onset of FN

- Very low MASCC risk-index score $(<15)$

MASCC Attribute ${ }^{a} \quad$ Score

o Burden of illness

- No or mild symptoms

- Moderate symptoms

- Severe symptoms

o No hypotension

o No COPD

o No previous fungal

infection

o No dehydration

o Outpatient status at onset of fever

o Age $<60$ yr

core

5
3
0
5
4
4
3
3
2

- Hypotension (SBP < $90 \mathrm{~mm} \mathrm{Hg}$ )

- Tachypnea (RR > 24)

- Serum albumin level $<3.3 \mathrm{~g} / \mathrm{dL}$

- Serum bicarbonate level $<21 \mathrm{mmol} / \mathrm{L}$

- C-reactive protein level $>20 \mathrm{mg} / \mathrm{L}$ at baseline

- High procalcitonin level (> $2.0 \mathrm{ng} / \mathrm{mL}$ )

- Circulating sTREM-1 > $100 \mathrm{pg} / \mathrm{mL}^{\mathrm{b}}$

- High PTX3 levels at the onset of $\mathrm{FN}^{\mathrm{b}}$

General factors associated with poor-prognosis FN

- Documented respiratory infection

- C-reactive protein level $>100 \mathrm{mg} / \mathrm{L}$ at day 5 of treatment for $\mathrm{FN}$

- Neutropenia lasting more than 4 days after an episode of FN

- Concurrent mucositis/colitis/typhlitis

- Intensive care unit admission

- Disseminated intravascular coagulation

- Confusion or altered mental status

- Bleeding severe enough to require transfusion

- Arrhythmia or ECG changes requiring treatment

Note. FN = febrile neutropenia; MASCC = Multinational Association of Supportive Care in Cancer; COPD = chronic obstructive pulmonary disease; SBP = systolic blood pressure; $\mathrm{RR}=$ respiration rate; $\mathrm{sTREM}-1=$ soluble triggering receptor; $\mathrm{PTX} 3=$ pentraxin $3 ; \mathrm{ECG}=$ electrocardiogram. Information from Ahn \& Lee (2012), Park et al. (2010), and Klastersky et al. (2007). aThe MASCC scoring system is intended for use in lowrisk FN. bNovel markers currently in early clinical trials.

Once cultures are obtained, the first priority is to administer IV antibiotics based on institu- 
tional policy, given variations in common microbial profiles by institution and by region (Klastersky et al., 2011). In the study conducted by Park et al. (2010), the most common first choice of antibiotics administered was cefepime (142 episodes, $55 \%$ ). The addition of a glycopeptide (e.g., vancomycin) for sustained fevers after 3 days of empiric antibiotics was most common, with the addition of antifungal agents based on suspected fungal etiology (Klastersky et al., 2011).

Factors associated with poor outcomes based on univariate analysis of the $259 \mathrm{FN}$ episodes included age $(p=.010)$ and comorbidities such as hypertension $(p=.012)$ and liver disease $(p=.029)$. The leading cause of death $(n=61[24 \%])$ was septic shock with multiorgan failure (21 cases) and respiratory failure ( 9 cases). Patients who developed a fever outside the hospital experienced more serious complications $(p=.003)$, emphasizing the need to have established protocols and effective patient and caregiver communication to expedite the management of $\mathrm{FN}$ in patients being cared for in the outpatient setting, particularly older patients. Recovery from neutropenia was found to be the most significant factor for survival $(p<.0001)$. Additional factors associated with poor-prognosis FN are included in Table 5.

In general, risk analysis based on the concepts previously discussed, incorporation of prophylactic administration of myeloid growth factors in high-risk populations based on consensus guidelines, and strategies for early identification and prompt initiation of treatment of FN are the backbone of managing CIN. Recommendations for the prevention and treatment of CIN and FN are summarized in Table 6.

Once a patient has experienced an episode of grades 3/4 CIN or FN, several additional considerations in continuing treatment come into play. Unlike patients who experience milder CIN (grades $1 / 2$ ), patients with more severe episodes will require greater scrutiny. The decision to resume treatment is based on the individual patient; his or her risk profile including underlying disease; the treatment regimen including therapeutic intent; and the availability of supportive care including growth factor support, accessibility to the clinical setting, financial resources, and caregiver support. Dose modification and/or dose delays may be required in patients with metastatic or incurable disease who may require treatment over extended periods of time.
The use of G-CSF as secondary prophylaxis has also been studied. Guidelines proposed by ASCO and the NCCN suggest the use of G-CSF in patients receiving mild to moderately myelotoxic chemotherapy with curative intent who have experienced an episode of grades 3/4 CIN or FN (Crawford et al., 2011; Aapro et al., 2011). Myeloid growth factors are not without potential AEs, the most common being bone pain (Table 6). Bone pain associated with G-CSF is generally self-limiting but may be severe (Wingard \& Elmongy, 2009). The administration of an anti-inflammatory agent such as naproxen together with an antihistamine such as loratadine at the onset of bone pain and continued for 48 to 72 hours works well to reduce the pain, with little risk of toxicity and minimal cost to the patient.

The use of prophylactic antibiotics remains controversial owing to limited data substantiating their benefit in the general cancer population together with concerns for the development of drug-resistant bacteria. Unlike myeloid growth factors, which have proven efficacy in preventing the incidence and severity of neutropenia, including episodes of $\mathrm{FN}$, prophylactic antibiotics serve to reduce the complications of neutropenic fevers (Wingard \& Elmongy, 2009). More recent trials have documented a reduction in the incidence of neutropenic fevers and bacteremia and infection-related mortality with administration of fluoroquinolones (ciprofloxacin and levofloxacin most common) in patients with hematologic or solid tumors (Wingard \& Elmongy, 2009; Wood \& Payne, 2011). Fluoroquinolones provide excellent coverage against Pseudomonas aeruginosa (ciprofloxacin) and Streptococcus (levofloxacin).

The concern for antibiotic-associated AEs, additional cost, secondary infection with Clostridium difficile, and the emergence of drug-resistant bacteria must be considered. However, prophylactic antibiotics in patients with hematologic malignancies or in patients at high risk for poorprognosis FN should be considered. Combining prophylactic antibiotics with G-CSF, in particular pegfilgrastim if the schedule permits, may provide the best option for prevention in very high-risk patients (Wingard \& Elmongy, 2009).

\section{Clinical Implications of Anemia}

Anemia is a common finding in patients with cancer, with an incidence ranging from $30 \%$ to 90\% (Rodgers et al., 2012). The causes of anemia 
Table 6. Recommendations for Prevention and Management of Chemotherapy-Induced Neutropenia and Febrile Neutropenia

\begin{tabular}{|c|c|}
\hline Assessment of risk & $\begin{array}{l}\text { - See Tables } 2 \text { and } 5 \text { for a description of risk factors for CIN due to disease- } \\
\text { related, host-related, and treatment-related factors. }\end{array}$ \\
\hline \multirow[t]{8}{*}{ Prevention } & $\begin{array}{l}\text { - Patient and caregiver education for infection prevention appropriate to th } \\
\text { level of risk }\end{array}$ \\
\hline & - Prophylactic use of colony-stimulating factors is recommended when: \\
\hline & $\begin{array}{l}\text { Risk of CTC-AE grades } 3 / 4 \mathrm{CIN} \text { or } \mathrm{FN} \text { is }>20 \% \text { in the setting of potentially } \\
\text { curable disease or where dose intensity is necessary for optimal clinical } \\
\text { outcomes. }\end{array}$ \\
\hline & $\begin{array}{l}\text { Risk of CTC-AE grades } 3 / 4 \text { CIN or FN is } 10 \%-20 \% \text { in patients with high } \\
\text { risk profile (Tables } 2 \text { and } 5 \text { ). }\end{array}$ \\
\hline & - FDA-approved agents: \\
\hline & o Filgrastim (Neupogen: See dosing guidelines at www.neupogen.com) \\
\hline & o Pegfilgrastim (Neulasta: See dosing guidelines at www.neulasta.com) \\
\hline & $\begin{array}{l}\text { - Consider prophylactic antibiotics for patients with hematologic } \\
\text { malignancies at very high risk for FN - fluoroquinolone +/- glycopeptide, } \\
\text { antifungal, antiviral. }\end{array}$ \\
\hline \multirow[t]{5}{*}{ Management of CIN } & - Implement primary prevention as above. \\
\hline & $\begin{array}{l}\text { - Establish a plan for close monitoring of blood counts in initial phase of } \\
\text { treatment where risk is greatest. }\end{array}$ \\
\hline & $\begin{array}{l}\text { - Review reportable signs and symptoms with patient and caregivers, } \\
\text { including who to contact and how to do so. }\end{array}$ \\
\hline & $\begin{array}{l}\text { - Subsequent treatment may require dose modification, dose delay, or } \\
\text { administration of G-CSF agents as secondary prophylaxis. }\end{array}$ \\
\hline & $\begin{array}{l}\text { - Low-risk patients with anticipated early recovery can be managed in an } \\
\text { outpatient setting. }\end{array}$ \\
\hline
\end{tabular}

- Most common AEs associated with G-CSF agents include bone pain, myalgia, arthralgia, and fever.

o Bone pain can be effectively managed with naproxen $225 \mathrm{mg}$ and loratadine $10 \mathrm{mg}$ q12 $\mathrm{h}$ at the onset of bone pain and continued until resolved (generally $48-72 \mathrm{hr}$ ).

Management of FN

- Considered a medical emergency

- Prompt intervention is critical to avoid morbidity and mortality.

- Rapid assessment for risk of clinical deterioration (Table 5)

- Implement institutional standard of care for FN, including obtaining cultures (blood and urine), PA and lateral chest x-ray, viral and VRE swabs if indicated, and prompt administration of IV antibiotics (cefepime most common first-line agent).

- Unstable patients should be transported by emergency medical services equipped with ACLS capabilities.

- Patients at very high risk for poor-prognosis FN may require ICU admission.

Note. $\mathrm{CIN}=$ chemotherapy-induced neutropenia; FN = febrile neutropenia; CTC-AE = Common Terminology Criteria for Adverse Events; FDA = US Food and Drug Administration; G-CSF = granulocyte colony-stimulating factor; $A E$ = adverse event; PA = posteroanterior; VRE = vancomycin-resistant enterococci; ACLS = advanced cardiac life support; ICU = intensive care unit. Information from Crawford et al. (2011), Aapro et al. (2011), Klastersky et al. (2011), Aapro, Crawford, \& Kamioner (2010), Wingard \& Elmongy (2009), and Talcott et al. (2011). 
in cancer patients vary widely, including metabolic and nutritional causes, chronic disease, renal insufficiency, blood loss, inadequate production due to bone marrow disease, peripheral destruction due to autoimmune disorders, druginduced red cell aplasia, and chemotherapy-induced anemia (CIA). Anemia is multifactorial in most cancer patients due to the generally older age of the cancer population, the existence of comorbidities with associated medications, and the effects of the malignancy and treatment (Rodgers et al., 2012). Chemotherapy-induced anemia is far less common than CIN, in part due to the difference of the life cycle of red blood cells; however, chemotherapeutic agents may contribute to anemia through disruption of normal hematopoiesis and interference of the cytokine milieu (Crawford et al., 2011).

The primary approach to the patient with anemia is as follows: (1) Establish the underlying cause(s), (2) treat the underlying cause(s), (3) evaluate symptoms of anemia with consideration of individual patient characteristics, and (4) weigh the risks and benefits of each treatment approach. The comprehensive assessment and management of anemia in the patient with cancer is beyond the scope of this article. However, supportive care for those receiving myelotoxic chemotherapy is an essential component of clinical management for these patients, including the treatment of anemia. Beyond the treatment of comorbid conditions and other contributing factors, the primary interventions for CIA include therapeutic transfusion of packed red blood cells (PRBCs) and administration of erythropoiesis-stimulating agents (ESAs; Rodgers et al., 2012).

TheFDA, the AABB, and the NCCN have recently revised the guidelines for administration of ESAs and PRBCs based on the specific risks and benefits of each approach (Carson et al., 2012; Vlaar, et al., 2011; Rodgers et al., 2012). Familiarity with these very recent recommendations is critical for the AP to provide informed consent to the patient, to maintain safety, and to ensure compliance with regulatory requirements, including the Risk Evaluation and Mitigation Strategy (REMS) program for erythropoiesis-stimulating agents regulated by the FDA and institutional blood bank requirements (Table 7).

Consideration of the individual patient characteristics and associated risks of PRBC transfusion and ESA administration is necessary. Thresholds for transfusion in particular will vary according to patient age, comorbidities, underlying disease, expected capacity for hematologic recovery, and ongoing therapy. It must always be kept in mind that the benefits of any transfusion are temporary, and restoration of normal bone marrow function and erythropoiesis is the most desirable goal. As noted in the REMS guidelines, there are specific risks associated with the administration of ESAs, including thrombosis, inferior survival, and decreased time to progression in selected tumor types. Thus, ESAs are reserved for patients receiving chemotherapy where treatment does not have a curative intent (Rodgers et al., 2012).

\section{Clinical Implications of Thrombocytopenia}

Thrombocytopenia and the risk of bleeding present a particular challenge in cancer patients undergoing cancer treatment. Like anemia, the cause may be multifactorial, including underlying disease, comorbidities, associated medications, disease-related destruction, or as a direct effect of treatment on the macrophages in the mitotic pool. Unlike neutropenia or anemia, the use of colony-stimulating factors for chemotherapy-induced thrombocytopenia is not yet FDA approved, in part due to concerns for off-target effects of thrombopoietic agents. The lifespan of a mature platelet in the postmitotic pool may be as little as $\mathbf{2 4}$ hours, thus there is a constant need for replacement. Patients with bone marrow disorders, continued peripheral destruction, or sequestration such as with splenomegaly are at particular risk for sustained thrombocytopenia. The risk of bleeding is the greatest concern for these patients. Criteria for therapeutic or prophylactic platelet transfusions, the type of platelets, and volume transfused have been reviewed (Slichter et al., 2010; Triulzi et al., 2012).

The Platelet Dose Study (PLADO), a database analysis of 3,447 hematology/oncology patients who received therapeutic platelet transfusions, found a platelet count of $10,000 / \mu \mathrm{L}$ to be the recommended trigger for prophylactic transfusion, with adaptation for patients with complicating factors, consistent with previous trials and consensus guidelines (Triulzi et al., 2012; Schiffer et al., 2001; Slichter, 2007; Slichter et al., 2010); see Table 8 . A platelet count $\geq 5,000 / \mu \mathrm{L}$ is thought to be sufficient to maintain endothelial integrity, a key factor in spontaneous bleeding risk. Educat- 


\section{Table 7. Recommendations for Management of Chemotherapy-Induced Anemia}

\section{Assessment of risk}

Patients at high risk for more serious complications of anemia include those with:

Cardiopulmonary disease, progressive or rapid decline in $\mathrm{Hgb}$ with or without recent chemotherapy or radiation,

Sustained symptoms of tachycardia, tachypnea, chest pain, dyspnea, syncope, debilitating fatigue

\section{Treatment of CIA}

General principles of treatment

- Establish the underlying cause(s): bleeding, nutritional, inherited, renal insufficiency, treatment, chronic disease, hemolysis

- Treat the underlying cause(s)

- Evaluate symptoms of anemia with consideration of individual patient characteristics

- Weigh the risks and benefits of each treatment approach (PRBC transfusion, ESA administration)

\section{Transfusion of PRBCs}

- Requires informed consent

- Asymptomatic patients: transfuse to maintain $\mathrm{Hgb} 7-9 \mathrm{~g} / \mathrm{dL}$

- Symptomatic with hemorrhage: transfuse to maintain hemodynamic stability

- Symptomatic with $\mathrm{Hgb}<10 \mathrm{~g} / \mathrm{dL}$ : transfuse to maintain $\mathrm{Hgb} 8-10 \mathrm{~g} / \mathrm{dL}$

- Acute coronary syndromes with anemia: transfuse to maintain $\mathrm{Hgb}>10 \mathrm{~g} / \mathrm{dL}$

Benefits

Rapid increase in Hgb may improve fatigue in some patients

Risks

- Viral transmission: HIV: 3.1/100,000, hepatitis C: 5.1/100,000, hepatitis B: 3.41-3.43/100,000

- Transfusion-related acute lung injury (TRALI): 0.81/100,000

- Transfusion-associated circulatory overload (TACO): 1\%-6\%, higher in ICU and postoperative settings

- Fatal hemolysis: 1.3-1.7/million transfused units

- Febrile nonhemolytic reactions: $1.1 \%-2.15 \%$

\section{ESA administration}

- FDA-approved agents: darbepoetin alfa (Aranesp), epoetin alfa (Epogen, Procrit)

- Not indicated in patients receiving chemotherapy with curative intent

- Requires REMS compliance and training for providers (ESA APPRISE Oncology Program)a

- Requires informed consent for patients

- Goal is to administer the lowest dose necessary to avoid PRBC transfusion not to exceed a $\mathrm{Hgb}$ of $10 \mathrm{~g} / \mathrm{dL}$

- If $\mathrm{Hgb}$ rises $>1 \mathrm{~g} / \mathrm{dL}$ in any 2-wk period, dose reductions are required; see prescribing information

Benefits

Avoidance of transfusions

Risks

- Inferior survival and decreased time to progression, most notably with target $\mathrm{Hgb}>12 \mathrm{~g} / \mathrm{dL}^{\mathrm{b}}$

- Thrombosis: increased risk history of coagulopathy, obesity, coronary artery disease, thrombocytosis,

hypertension, immobilization, hospitalization, selected hormonal therapies, immunomodulatory agents ${ }^{c}$

- Hypertension/seizures

- Pure red cell aplasia (rare)

Note. $\mathrm{Hgb}$ = hemoglobin; $\mathrm{CIA}=$ chemotherapy-induced anemia; PRBC = packed red blood cell; ESA = erythropoiesisstimulating agent; ICU = intensive care unit; FDA = US Food and Drug Administration; REMS = Risk Evaluation and Mitigation Strategy. Information from Carson et al. (2012), Vlaar et al. (2011), and Rodgers et al. (2012).

ahttps://www.esa-apprise.com/ESAAppriseUI/ESAAppriseUI/default.jsp

bhttp://www.fda.gov/cder/drug/infopage/RHE/default.htm

chttp://www.nccn.org/professionals/physician_gls/f_guidelines.asp\#supportive

ing the patient and their caregivers about bleeding precautions and reportable signs and symptoms is critical to avoiding more serious adverse events.

\section{Summary and Conclusions}

Chemotherapy-induced myelotoxicity is a common and potentially life-threatening adverse event for cancer patients. Neutropenia, febrile neutropenia, anemia, and thrombocytopenia are the result of complex processes as a result of the disease, the approach to treatment, and the characteristics of the individual patient. Assessment of individual risk using the criteria described, implementing prevention, monitoring, and treatment strategies, as well as setting expectations for the patient and family are essential to avoid more serious adverse events. Familiarity with the risks and benefits of supportive care measures for the treatment of cytopenias, recent up- 


\section{Table 8. Recommendation for Management of Chemotherapy-Induced Thrombocytopenia}

\section{Assessment of risk}

- CTCAE risk (Table 4) and WHO bleeding grades

1. Petechiae, ecchymosis, occult blood in body secretions, mild vaginal spotting

2. Evidence of gross hemorrhage not requiring red blood cell transfusion over routine needs: epistaxis, hematuria, hematemesis

3. Hemorrhage of one or more units of PRBCs/day

4. Life-threatening hemorrhage, defined as either massive bleeding causing hemodynamic compromise or bleeding into a vital organ (e.g., intracranial, pericardial, or pulmonary hemorrhage)

- Evaluate symptoms and underlying disease

- Determine chronicity

- Consider individual characteristics of the patients, including proximity to treatment center, concomitant anticoagulation therapy or antiplatelet drugs, prior response to platelets, concurrent inflammatory process/infection, CNS disease

\section{Prevention}

- Evaluate bleeding risk as above

- Establish plan of care for monitoring blood counts and follow-up

- Maintain a current type and screen blood identification band for patients requiring frequent transfusions

- Hold anticoagulation therapy for platelet count $<50,000 / \mu \mathrm{L}$

- Educate the patient and caregivers about bleeding precautions and reportable signs and symptoms

\section{Platelet products}

- Random-donor platelets

o Centrifuged from whole blood

o Common dose administered is 4-6 random-donor units (pooled from multiple units of whole blood)

o Larger volume: 1 unit $=60 \mathrm{~mL}, 6$ units $=360 \mathrm{~mL}$

- Exposure to more donors

- Single-donor platelets

o Single donor undergoes apheresis, process requires $1.5-2.5 \mathrm{hr}$

o Approximately $200 \mathrm{~mL}$

o Process is costly, more than twice the cost of random-donor platelets

o More recent procedures produce leukoreduced product

\section{Platelet transfusions based on platelet levels and patient characteristics}

- Platelets $<10,000 / \mu \mathrm{L}$, threshold for therapeutic platelet transfusions

- Patients with a history of bleeding or an active infectious process may require higher threshold for transfusion

- Surgical or invasive procedure: maintain platelets $>50,000 \mu \mathrm{L}$, more aggressive procedures for patients with complicating factors may require higher platelet count

- Neurosurgical procedures: maintain platelets $>100,000 \mu \mathrm{L} / \mathrm{L}$

- Premedication for platelets may be required in patients with history of urticaria

- Evaluate response to platelets by obtaining postplatelet count 30-60 min after infusion

Note. CTCAE = Common Terminology Criteria for Adverse Events; WHO = World Health Organization; PRBC $=$ packed red blood cell; CNS = central nervous system. Information from Slichter (2007), Triulzi et al. (2012), Slichter et al. (2010), Schiffer et al. (2001), and Kurtin et al. (2012).

dates to consensus guidelines, recommendations for treatment modification, and contraindications for various supportive care measures will assist the AP in oncology in effectively managing chemotherapyinduced myeloid toxicity.

\section{DISCLOSURE}

The author has no conflicts of interest to disclose.

\section{REFERENCES}

Aapro, M. S., Bohlius, J., Cameron, D. A, Dal Lago, L., Donnelly, J. P., Kearney, N.,...Zielinski, C. (2011). 2010 update of EORTC guidelines for the use of granulocyte-colony stimulating factor to reduce the incidence of chemotherapy-induced febrile neutropenia in adult patients with lymphoproliferative disorders and solid tumors. European Journal of Cancer, 47, 8-32. http://dx.doi.org/10.1016/j. ejca.2010.10.013

Aapro, M., Crawford, J., \& Kamioner, D. (2010). Prophylaxis of chemotherapy-induced febrile neutropenia with granulocyte-stimulating factors: Where are we now? Supportive Care in Cancer, 18, 529-541. http://dx.doi.org/10.1007/ s00520=010-0816-y

Ahn, S., \& Lee, Y. S. (2012). Predictive factors for poor prognosis febrile neutropenia. Current Opinion in Hematology, 24, 376-380.

Barr, P. J., Donnelly, M., Cardwell, C. R., Parker, M., Morris, K., \& Bailie, K. E. (2011). The appropriateness of red blood cell use and the extent of overtransfusion: Right decision? Right amount? Tranfusion, 51, 1684-1694. 10.1111/j.15372995.2011.03130.x

Bejar, R., Levine, R., \& Ebert, B. L. (2011). Unraveling the molecular pathophysiology of myelodysplastic syndromes. Journal of Clinical Oncology, 29, 504-515. http://dx.doi. 
org/10.1200/JCO.2010.31.1175

Carlesso, N., \& Cardoso, A. (2010). Stem cell regulatory niches and their role in normal and malignant hematopoiesis. Current Opinion Hematology, 17, 281-286. http://dx.doi. org/10.1097/MOH.0b013e32833a25d8

Carreca, I., \& Balducci, L. (2009). Cancer chemotherapy in the older patient. Urologic Oncology, 27, 633-642. http:// dx.doi.org/10.1016/j.urolonc.2009.08.006

Carson, J. L., Grossman, B. J., Kleinmann, S., Tinmouth, A. T., Marques, M. B., Fung, M. K., Holcomb, J. B.,...Djulbegovic, B. (2012). Red blood cell transfusion: A clinical practice guideline from the AABB. Annals of Internal Medicine, E-pub March 26, E-429 http://dx.doi.org/10.7326/00034819-156-12-201206190-00429

Catenacci, D. V., \& Schiller, G. J. (2005). Myelodysplastic syndromes: A comprehensive review. Blood Reviews, 19, 301319. http://dx.doi.org/10.1016/j.blre.2005.01.004

Chan, A., Verma, S. Loibl, S., Crawford, J., Choi, M. R., Dreiling, L., \& Vandenberg, T. (2011). Reporting of myelotoxicity associated with emerging regimens for the treatment of selected solid tumors. Critical Reviews in Oncology Hematology, 81, 136-150. http://dx.doi.org/10.1016/j.critrevonc.2011.03.003

Corey, S. J., Minden, M. D., Barber, D. A., Kantarjian, H., Wang, J. C. Y., \& Schimmer, A. D. (2007). Myelodysplastic syndromes: The complexity of stem-cell diseases. Nature Reviews Cancer, 7, 118-129. http://dx.doi.org/10.1038/nrc2047

Crawford, J., Allen, J., Armitage, J., Blayney, D. W., Cataland, S. R., Heanwey, M. L., Htoy, S., \& Westmoreland, M. (2011). Myeloid growth factors. Journal of the National Comprehensive Cancer Network, 9, 914-932.

Crea, F., Giovannetti, E., Zinzani, P. L., \& Danesi, R. (2009). Pharmacological rationale for early G-CSF prophylaxis in cancer patients and role of pharmacogenetics in treatment optimization. Critical Reviews in Oncology Hematology, 72, 21-44. http://dx.doi.org/10.1016/j.critrevonc.2008.10.014

Daniel, D., \& Crawford, J. (2006). Myelotoxicity from chemotherapy. Seminars in Oncology, 33, 74-85. http://dx.doi. org/10.1053/j.seminoncol.2005.11.003

Flores, I. Q., \& Ershler, W. (2009). Managing neutropenia in older patients with cancer receiving chemotherapy in a community setting. Clinical Journal of Oncology Nursing, 14, 81-86. http://dx.doi.org/10.1188/10.CJON.81-86

Gatter, K., Natkunam, Y., \& Brown, D. (2008). Bone marrow diagnosis: An illustrated guide (2nd ed., p. 6). Hoboken, NJ: Wiley-Blackwell.

Glaspy, J., Crawford, J., Vansteenkiste, J., Henry, D., Rao, S., Bowers, P., Berlin, J. A., Tomita, D., Bridges, K., \& Ludwig, H. (2010). Erythropoeisis-stimulating agents in oncology: A study-level meta-analysis of survival and other safety outcomes. British Journal of Cancer, 102, 301-315. http:// dx.doi.org/10.1038/sj.bjc.6605498

Greipp, P. R., SanMiguel, J., Durie, B. G., Crowley, J. J., Barlogie, B., Bladé, J.,...Westin, J. (2005). International staging system for multiple myeloma. Journal of Clincial Oncology, 23(15), 3412-3420. http://dx.doi.org/10.1200/JCO.2005.04.242

Hadland, B. K., \& Longmore, G. D. (2009). Erythroid-stimulating agents in cancer therapy: Potential dangers and biological mechanisms. Journal of Clinical Oncology, 27, 42174226. http://dx.doi.org/10.1200/JCO.2008.21.6945

Klastersky, J., Awada, A., Paesmans, M., \& Aoun, M. (2011). Febrile neutropenia: A critical review of the initial management. Critical Reviews in Oncology Hematology, 78, 185194. http://dx.doi.org/10.1016/j.critrevonc.2010.03.008

Klastersky, J., Ameye, L., Maertens, J., Georgala, A., Muanza, F., Aoun, M.,...Paesmans, M. (2007). Bacteraemia in fe- brile neutropenic cancer patients. International Journal of Antimicrobial Agents, 30(suppl 1), S51-S59. http://dx.doi. org/10.1016/j.ijantimicag.2007.06.012

Kurtin, S. (2010). Risk analysis in the treatment of hematologic malignancies in the elderly. Journal of the Advanced Practitioner in Oncology, 1, 119-129.

Kurtin, S. (2011a). Current approaches to the diagnosis and management of myelodysplastic syndromes. Journal of the Advanced Practitioner in Oncology, 2(suppl 2), 7-18.

Kurtin, S. (2011b). Leukemia and myelodysplastic syndromes. In C. H. Yarbro, D. Wujcik, \& B. H. Gobel (Eds.), Cancer nursing: Principles and practice (7th ed., pp. 1369-1398). Sudbury, MA: Jones and Bartlett.

Kurtin, S. E., Demakos, E., Hayden, J., \& Boglione, C. (2012). Initial treatment of myelodysplastic syndromes: Practical tools and effective management. Clinical Journal of Oncology Nursing, 16(3 suppl 1), 23-35. http://dx.doi. org/10.1188/12.CJON.S1.23-35

Ludwig, H., \& Zojer, N. (2008). Recent therapeutic advances in hematological malignancies: Dealing with treatmentrelated complications. Annals of Oncology, 19(suppl 5), v71-v78. http://dx.doi.org/10.1093/annonc/mdn315

Lyman, G., Dale, D. C., Wolff, D. A., Culakova, E., Poniewierski, M. S., \& Kuderer, N. M. (2010). Acute myeloid leukemia or myelodysplastic syndrome in randomized controlled clinical trials of cancer chemotherapy with granulocyte colony-stimulating factor: A systematic review. Journal of Clinical Oncology, 28, 2914-2924. http://dx.doi.org/10.1200/ JCO.2009.25.8723

Maxwell, M. B., \& Maher, K. E. (1992). Chemotherapy-induced myelosuppression. Seminars in Oncology Nursing, 8, 113123. http://dx.doi.org/10.1016/0749-2081(92)90027-Z

Metcalf, D. (2010). The colony stimulating factors and cancer. Nature Reviews Cancer, 10, 425-434. http://dx.doi. org/10.1038/nrc2843

Miyoshi, N., Yano, M., Takachi, K., Kishis, K., Noura, S., Eguchi, W.,...Imaoka, S. (2009). Myelotoxicity of preoperative chemoradiotherapy is a significant determinant of poor prognosis in patients with $\mathrm{T} 4$ esophageal cancer. Journal of Surgical Oncology, 99, 302-306. http://dx.doi. org/10.1002/jso.21235

National Comprehensive Cancer Network. (2011). NCCN Clinical Practice Guidelines in Oncology. Prevention and treatment of cancer-related infections. Version 1.2011. National Comprehensive Cancer Network. Retrieved from http:// www.ncen.org

National Institutes of Health. (2012). Stem cell basics. Retrieved from http://stemcells.nih.gov/info/basics/basics4.asp

Park, Y., Kim, D. S., Park, S. J., Seo, H. Y., Lee, S. R., Sung, H. J.,... Kim, J. S. (2010). The suggestion of a risk stratification system for febrile neutropenia with hematologic disease. Leukemia Research, 34, 294-300. http://dx.doi.org/10.1016/j. leukres.2009.08.024

Rodgers, G. M., Becker, P. S., Blinder, M., Cella, D., ChananKhan, A., Cleeland, C.,...Wier, A. B. (2012). Cancer and chemotherapy-induced anemia. Journal of National Comprehensive Cancer Network, 10, 628-653.

Schiffer, C. A., Anderson, K., Bennett, C. L., Bernstein, S., Elting, L. S., Goldsmith, M., Goldstein, M.,...Wagnon, A. H. (2001). Platelet transfusion for patient with cancer: Clinical Practice Guidelines of the American Society of Clinical Oncology. Journal of Clinical Oncology, 19, 1519-1538.

Schwenkglenks, M., Pettengell, R., Jackisch, C., Paridaens, R., Constenal, M., Bosly, A.,...Leonard, R. (2011). Risk factors for chemotherapy-induced neutropenia occurrence in breast cancer patients: Data from the INC-EU Prospective 
Observational European Neutropenia Study. Supportive Care in Cancer, 19, 483-490. http://dx.doi.org/10.1007/ s00520-010-0840-y

Scripture, C. D., \& Figg, W. D. (2006). Drug interactions in cancer therapy. Nature Reviews Cancer, 6, 546-559. http:// dx.doi.org/10.1038/nrc1887

Sekeres, M. A. (2011). Epidemiology, natural history, and practice patterns of patients with myelodysplastic syndromes in 2010. Journal of the National Comprehensive Cancer Network, 9, 57-63.2011

Singbrant, S., Russell, M. R., Jovic, T., Liddicoat, B., Izon, D. J., Purton, L. E.,...Walkley, C. R. (2011). Erythropoietin couples erythropoiesis, B-lymphopoiesis, and bone homeostasis within the bone marrow microenvironment. Blood, 117, 5631-5642. http://dx.doi.org/10.1182/ blood-2010-11-320564

Slichter, S., Kaufman, R. M., Assmann, S. F., McCullough, J., Triulzi, D. J., Strauss, R. G.,...Granger, S. (2010). Dose of prophylactic platelet transfusions and prevention of hemorrhage. New England Journal of Medicine, 362, 600-613. http://dx.doi.org/10.1056/NEJMoa0904084

Slichter, S. J. (2007). Evidence-based platelet transfusion guidelines. Hematology, 2007(1), 172-178. http://dx.doi. org/10.1182/asheducation-2007.1.172

Smith, R. E. (2006). Trends in recommendations for myelosuppressive chemotherapy for the treatment of solid tumors. Journal of the National Comprehensive Cancer Network, 4, 649-658.

Talcott, J. A., Yeap, B. Y., Clark, J. A., Siegel, R. D., Loggers, E. T., Lu, C., \& Godley, P. A. (2011). Safety of early discharge for low-risk patients with febrile neutropenia: A mul- ticenter randomized controlled trial. Journal of Clinical Oncology, 29, 3977-3983. http://dx.doi.org/10.1200/ JCO.2011.35.0884

Triulzi, D. J., Assmann, S. F., Strauss, R. G., Ness, P. M., Hess, J. R., Kaufman, R. M....Slichter, S. (2012). The impact of platelet transfusion characteristics on post-transfusion platelet increments and clinical bleeding in patients with hypoproliferative thrombocytopenia. Blood, 119, 5553-5562. http:// dx.doi.org/10.1182/blood-2011-11-393165

Undevia, S. D., Gomez-Abuin, G., \& Ratain, M. (2005). Pharmacokinetic variability of anticancer agents. Nature Reviews Cancer, 5, 447-458. http://dx.doi.org/10.1038/nrc1629

U. S. Department of Health \& Human Services. (2010). Common Terminology Criteria for Adverse Events (CTCAE) Version 4.0. National Institutes of Health, National Cancer Institute. Retrieved from http://evs.nci.nih.gov/ftpl/CTCAE_4.03_2010-06-14_Quickreference_5X7.pdf

Vlaar, A. P., Hofstra, J., Determann, R. M., Veelo, D. P., Paulus, F., Kulik, W.,...Juffermans, N. P. (2011). The incidence, risk factors, and outcome of transfusion-related acute lung injury in a cohort of cardiac surgery patients: A prospective nested case-control study. Blood, 117, 4218-4225. http:// dx.doi.org/10.1182/blood-2010-10-313973

Wingard, J. R., \& Elmongy, M. (2009). Strategies for minimizing complications of neutropenia: Prophylactic myeloid growth factors or antibiotics. Critical Reviews in Oncology Hematology, 72, 144-154. http://dx.doi.org/10.1016/j.critrevonc.2009.01.003

Wood, S., \& Payne, J. (2011). Cancer-related infections. Journal of the Advanced Practitioner in Oncology, 2, 356-371.

\section{Save the Date: October 11-14, 2012}

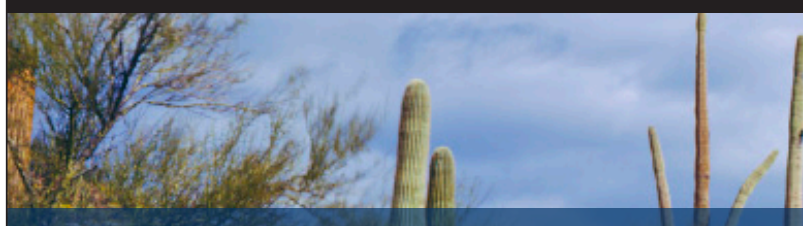

\section{5th Annual}

APAO Conference

\section{Best of ASC( including Best of ASCO $^{\circledR} 2012$}

Annual Meeting'12 *Official BEST OF ASCO Meeting, a program licensed and endorsed by the American Society of Clinical Oncology.
${ }^{*}$ Best of ASCO ${ }^{*}$ and any related logos are trademarks of the American Society of Clinical Oncology.

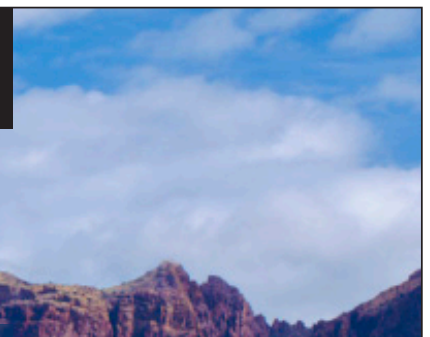

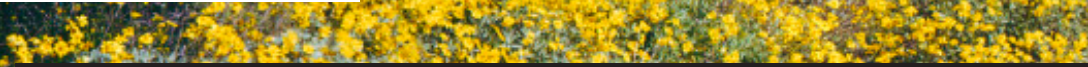

Hilton Scottsdale Resort and Villas • 6333 North Scottsdale Road • Scottsdale, Arizona

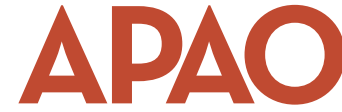

ASSOCIATION OF

PHYSICIAN ASSISTANTS IN ONCOLOGY

partners in cancer care
THE UNIVERSITY OF TEXAS

MDAnderson EancerCenter 